\title{
Três Visões Sobre Sustentabilidade e implicações para a avaliação em Ciência, Tecnologia e Inovação ${ }^{1}$
}

\author{
Three visions on sustainability and implications for Science, Technology and \\ Innovation evaluation
}

\author{
Rosana Icassatti Corazza ${ }^{2}$ \\ Maria Beatriz Machado Bonacelli ${ }^{3}$ \\ Paulo Sérgio Fracalanza ${ }^{4}$
}

\begin{abstract}
RESUMO
Inovação e sustentabilidade são temas amplos e complexos. Nos últimos anos, crescem as referências a termos como "inovação sustentável", "eco-inovação" e "inovação para a sustentabilidade". A sustentabilidade passa a ser atributo positivo e desejável do desenvolvimento científico, tecnológico e da inovação em si. Entretanto, a literatura especializada na temática da sustentabilidade abrange inúmeras perspectivas que guardam, em alguma medida, um forte parentesco com áreas disciplinares específicas, tanto nas ciências naturais quanto nas ciências sociais aplicadas. Se a afirmação de que uma iniciativa (seja ela uma nova tecnologia, seja um novo empreendimento) é sustentável implica que se avalie sua sustentabilidade, a etapa lógica anterior ao próprio desenvolvimento dos métodos de avaliação é a concepção do que vem a ser sustentabilidade. O objetivo precípuo deste artigo é apresentar três visões que emergem do estudo da literatura especializada no tema da sustentabilidade, apresentando elementos para sua análise. Lado a lado, identifica-se a emergência de um "campo interdisciplinar" voltado ao tema da sustentabilidade, e também uma importância destacada de algumas perspectivas disciplinares sobre o conceito central. Conclui-se pela necessidade de avanços na consolidação de uma perspectiva interdisciplinar que possa fundamentar os processos de avaliação.

Palavras-chave: Sustentabilidade. Triple bottom line. Resiliência. Capacidade suporte.
\end{abstract}

\begin{abstract}
Innovation and sustainability are broad and complex issues. In recent years, references are growing to terms like "sustainable innovation", "eco-innovation" and "innovation for sustainability." Sustainability becomes a positive and desirable attribute of scientific and technological development and innovation itself. However, the literature on the subject of sustainability encompasses many perspectives that keep to some extent, a strong affinity with specific subject areas, both in the natural sciences and in the applied social sciences. If the statement that an initiative (whether it be a new technology or a new business) is sustainable imply evaluate its sustainability, the logical step preceding the actual development of the evaluation methods is the conception of what sustainability is. The main objective of this article is to present conceptual views that emerge from the study of literature on the topic of sustainability, presenting elements for their analysis. Side by side, one identifies the emergence of an "interdisciplinary field" oriented the subject of sustainability and a pronounced importance of some disciplinary perspectives on the key concepts. One concludes by the need of consolidating an interdisciplinary perspective that can support the evaluation processes.
\end{abstract}

Keywords: Sustainability. Triple bottom line. Resilience. Carrying capacity.

\footnotetext{
${ }^{1}$ Trabalho apresentado no dia 17 de outubro de 2013, no Grupo de Trabalho 19 - Políticas Públicas de Ciência, Tecnologia e Inovação na América Latina: desafios e contribuições.

${ }^{2}$ Doutora em Política Científica e Tecnológica pelo Departamento de Política Científica e Tecnológica (DPCTUnicamp); Professora na Faculdades de Campinas (Facamp); Pesquisadora Colaboradora do DPCT/IG/Unicamp e do Núcleo de Economia Industrial e da Tecnologia (NEIT) - IE/Unicamp. E-mail: rosanacorazza@gmail.com

${ }^{3}$ Doutora em Economia pela Université de Toulouse; Professora e Coordenadora Geral dos Programas de Pós-Graduação do Instituto de Geociências (CPG) e Pesquisadora Associada do GEOPI (www.ige.unicamp.br/geopi). E-mail: bia@ige.unicamp.br

${ }_{4}^{4}$ Doutor em Economia pelo Instituto de Economia da Unicamp; Professor e Coordenador Geral dos Programas de Pós-Graduação do IE/Unicamp. Pesquisador do Núcleo de Economia Industrial e da Tecnologia (NEIT) - IE/Unicamp. E-mail: fracalan@eco.unicamp.br
} 


\section{INTRODUÇÃO}

Sustentabilidade tem se tornado um termo de uso absolutamente corrente em nossos dias. A pesquisa do termo "sustentabilidade" no mecanismo de busca Google origina aproximadamente onze milhões e meio de resultados; na versão do aplicativo em inglês, a palavra "sustainability" permite identificar 115 milhões. O termo é empregado nos mais diversos fóruns e arenas de debate. $\mathrm{Na}$ academia, encontram-se referências nos mais diversos campos disciplinares e também em estudos apresentados como interdisciplinares. Na esfera pública, é matéria de ações, projetos, programas e políticas governamentais nos níveis municipais, estaduais e federais. É empregado por empresas atuantes nos mais diversos setores, nas mais distintas escalas de operação, em atividades que vão do desenvolvimento de produtos e serviços ao marketing, passando por transformações dos processos produtivos, até a gestão de maneira ampla, incluindo supply chain, pessoal, inovação e relações organizacionais (Jabbour et al, 2013). No domínio do terceiro setor, o apelo à sustentabilidade deixou de se circunscrever à militância ambientalista. Dos papeis de environmental watchdogs, dos formadores de opinião e mobilizadores da ação pública, o terceiro setor passou a fortalecer papeis anteriormente menos conspícuos e assumir novas funções, como a execução de pesquisa, o desenvolvimento de comunidades dependentes de recursos naturais, a busca de recursos para financiamento dessas atividades, a educação para a sustentabilidade, dentre outros.

A Comissão Mundial para Meio Ambiente e Desenvolvimento das Nações Unidas se propunha, em 1988, a tratar dos conflitos entre proteção do meio ambiente e desenvolvimento. Sua definição de desenvolvimento sustentável é a mais conhecida e citada: "Desenvolvimento sustentável é aquele que satisfaz as necessidades do presente sem comprometer a capacidade de que as gerações futuras satisfaçam suas próprias necessidades". (CMMAD/ONU, 1988)

O aparente consenso que se estabelece desde então oculta, entretanto, uma diversidade de posturas e enfoques propugnados nos mais diferentes fóruns de tomada de decisão e em disciplinas acadêmicas particulares. No intuito de esclarecer algumas desses enfoques, Kemp (2010) empreendeu um breve levantamento consistente com a percepção de outros autores sobre a proliferação 
do uso do termo sustentabilidade a respeito do qual as interpretações são frouxas, quando não até mesmo conflitantes. Simões (2012) indica o registro de mais de 200 conceituações de "sustentabilidade".

A multiplicidade de conceitos que constata não se resume a uma frouxidão interpretativa ou a um uso excessivamente permissivo do termo. Decerto isso também ocorre, muitas vezes levando a uma sensação de esvaziamento do conteúdo; mas o que gostaríamos de apontar, aqui, são clivagens de interpretação que se relacionam a perspectivas construídas de modo mais consistentes, com a contribuição de ramos específicos do conhecimento, mais ou menos de maneira "disciplinar" ou "interdisciplinar". Neste sentido, destacamos três usos dentre os mais comuns: o primeiro deles no âmbito das Ciências Econômicas; o segundo, no âmbito das Ciências da Gestão ou da Administração; e o terceiro, no âmbito da Ciência da Sustentabilidade. Finalmente, apresentamos uma síntese e algumas implicações para os esforços em termos de avaliação da CT\&l.

\section{SUSTENTABILIDADE FORTE OU FRACA?}

No âmbito das Ciências Econômicas, é conhecida a dualidade da interpretação da sustentabilidade forte e da sustentabilidade fraca. Um país, um investimento, um projeto ou um negócio poderão ser fracamente sustentáveis se o que estiverem fazendo no momento permitir que as gerações futuras alcancem os mesmos padrões (atuais) de vida - ou padrões melhores. Em outras palavras, se permitir às gerações futuras "a satisfação de suas necessidades" (CMMAD/ONU, 1987). Esta seria a definição central, de acordo com Neumeyer (2010), de sustentabilidade da perspectiva da justiça diacrônica conforme enunciada pelo Relatório Brundtland.

Colocar a questão do bem-estar numa dimensão intergeracional não é exatamente uma dificuldade com a qual a Economia não se tenha defrontado anteriormente. Desde Hotelling (1931) a questão da exploração dos recursos naturais exauríveis conta com um arcabouço teórico-metodológico para o tratamento intertemporal, na qual a escolha do horizonte temporal da depleção é uma variável de escolha. O autor propôs um modelo para a determinação da taxa ótima de 
extração de recursos naturais não renováveis que ainda hoje é considerada um marco da abordagem econômica (neoclássica) para esses recursos. Do ponto de vista da poluição, o tratamento paradigmático das externalidades negativas e grande parte dos instrumentos econômicos para a internalização desses "custos externos" existem em suas linhas essenciais desde o trabalho de Pigou (1920). Embora técnicas como as taxas de desconto, a análise custo-benefício, o cálculo de custos externos, as técnicas de valoração econômica de custos e benefícios ambientais e assim por diante existam desde a primeira metade do século passado, as abordagens da sustentabilidade se desenvolvem mais recentemente no âmbito das Ciências Econômicas.

Ayres et al (1998) notam que a visão econômica sobre a sustentabilidade consiste num problema de gestão do portfólio de capital nacional a fim de mantê-lo constante ao longo do tempo. O capital da nação inclui tanto o capital natural quanto o capital feito pelo homem. Há duas perspectivas gerais sobre as possíveis relações entre essas duas formas de capital, entretanto: a de substitutibilidade e a de complementaridade. Embora se possa admitir, em casos particulares, graus intermediários de substitutibilidade ou de complementaridade, destacam-se os dois modelos a seguir.

O primeiro modelo corresponde à sustentabilidade fraca, que assume a substitutibilidade perfeita entre o capital natural e o capital feito pelo homem. Esta tem sido a visão defendida por economistas neoclássicos, como Solow $(1974,1986)$ e pode ser colocada nos seguintes termos: o valor total dos bens (produtos manufaturados e serviços) mais o capital natural deve permanecer constante ao longo do tempo, implicando que reduções neste último devam ser compensadas pelo aumento dos primeiros.

Em favor desta perspectiva, é preciso reconhecer que mesmo os teóricos desta linhagem têm reconhecido que é necessário levar em conta plenamente, nos sistemas de contabilidade nacional dos países, a depleção do capital natural. (Cf., por exemplo, El Serafy, 1993 e 1997).

É interessante observar duas críticas colocadas por Gowdy \& McDaniel (1999) a este modelo de sustentabilidade a partir de um caso ilustrativo estudado pelos autores. É o caso da pequena nação insular no Pacífico, Nauru, onde foram explorados os ricos depósitos mundiais de fosfato a partir de 1900. Depois de quase 
um século de exploração mineral, $80 \%$ do território de Nauru encontrava-se devastado. O país soube proteger os recursos oriundos da exploração mineral por meio de um fundo soberano de US\$ 1 bilhão, cujos rendimentos seriam suficientes para manter uma renda estável aos habitantes da ilha. Os autores lamentam que a crise financeira asiática de 1997 devastou o fundo soberano. A crítica, neste caso, reside no fato de que os recursos naturais, uma vez "financeirizados", tornam-se passíveis de perdas catastróficas em momentos de grande instabilidade e crises financeiras num mundo financeiramente globalizado e interdependente. As críticas dos autores ao modelo da sustentabilidade fraca consistem de duas "lições" extraídas do caso de Nauru. A primeira diz respeito à irreversibilidade: a substitutibilidade entre capital natural e capital feito pelo homem é unidirecional; uma vez que algo é convertido em capital manufaturado, não existe como voltar à situação original. A segunda "lição", que guarda relação com a primeira, é que a transformação de capital natural em capital financeiro coloca uma nova dimensão de risco à riqueza natural e ao desenvolvimento dela dependente. O caso de Nauru evidencia que um recurso natural fisicamente depletado e "transmutado" em forma financeira, sofre, tanto quanto quaisquer outras formas de capital financeiro, perdas que podem ser monumentais numa escala de tempo quase instantânea.

$\mathrm{Na}$ visão econômica, o modelo alternativo à sustentabilidade fraca é o de sustentabilidade forte que tem seu fundamento no reconhecimento de que a possibilidade de substituição entre o capital feito pelo homem e o capital natural é limitada, quando não inexistente; de fato, não é possível nenhuma exploração ou uso econômico de recursos naturais sem a existência desses mesmos recursos. (Ayres et al, 1998; Neumeyer, 2010). Nesta senda teórica, acredita-se que é necessário que se mantenha um mínimo de diferentes tipos de capital (que agora podem ser compreendidos em suas manifestações ecológicas, sociais e econômicas) em termos físicos. A primeira motivação para essa manutenção é o reconhecimento de que os recursos naturais são insumos essenciais para a produção, o consumo e o bem-estar (Ayres et al, 1998). Neste sentido advoga-se a necessidade não apenas de se identificar e mensurar os limites das reservas, mas também as capacidades de absorção e de resiliência dos ecossistemas. A segunda motivação para essa manutenção é, de acordo com Ayres et al (1998) é "quasimoral": o reconhecimento da integridade da natureza e do direito à vida das 
espécies. Os autores ainda lembram que as versões mais atuais, sobretudo da Economia Ecológica como Daly (1995) e seus seguidores, da sustentabilidade forte, focaliza que os ativos ambientais são críticos no sentido de prover serviços de suporte à vida insubstituíveis (Ayres et al, 1998:5).

A relação entre a sustentabilidade forte e a sustentabilidade fraca depende da elasticidade de substituição entre o capital natural e outras formas de capital, como o capital físico e o capital intelectual. Se a elasticidade de substituição for suficientemente baixa, os conceitos de sustentabilidade forte e de sustentabilidade fraca não serão significativamente diferentes. No limite, o capital natural e outros tipos de capital podem ser considerados complementares. Se a elasticidade de substituição for alta, os dois tipos de capital não são considerados significativamente distintos (no limite, serão substitutos perfeitos) e a manutenção dos níveis de bemestar econômico não requer a manutenção de outras espécies e de outras formas de riquezas naturais. (Neumeyer, 2010).

\section{0 "TRIPÉ" DA SUSTENTABILIDADE ORGANIZACIONAL}

A perspectiva mais amplamente abraçada pelas Ciências da Gestão ou Administração é a aquela conhecida como Triple Bottom Line, segundo a qual a sustentabilidade de uma organização - ou de um negócio - deveria ser avaliada de maneira mais ampla do que a convencional bottom line. A bottom line é a maneira informal de se referir à receita líquida, de modo que a bottom line de uma empresa se refere à sua receita total menos suas despesas operacionais, juros pagos, depreciação e taxas (Farlex Financial Dictionary, 2012). Também é possível fazer a relação desses resultados do ponto de vista de sua distribuição entre os acionistas (shareholders): aumentar a bottom line de uma empresa, neste sentido, significa aumentar os ganhos por ação (EPS na sigla em inglês para Earnings Per Share). John Elkington, propõe que se compreenda a "sustentabilidade" de uma empresa a partir de a perspectiva que, ao lado da bottom line convencional, também sejam ponderados os aspectos social e ambiental, daí as expressões "TBL - Triple Bottom Line", "3Ps - Profits, People \& Planet", "tripé da sustentabilidade" e "três pilares da sustentabilidade" pelas quais sua abordagem também é conhecida. 
A figura abaixo ilustra o conceito de "sustentabilidade organizacional" proposto por Elkington para as empresas.

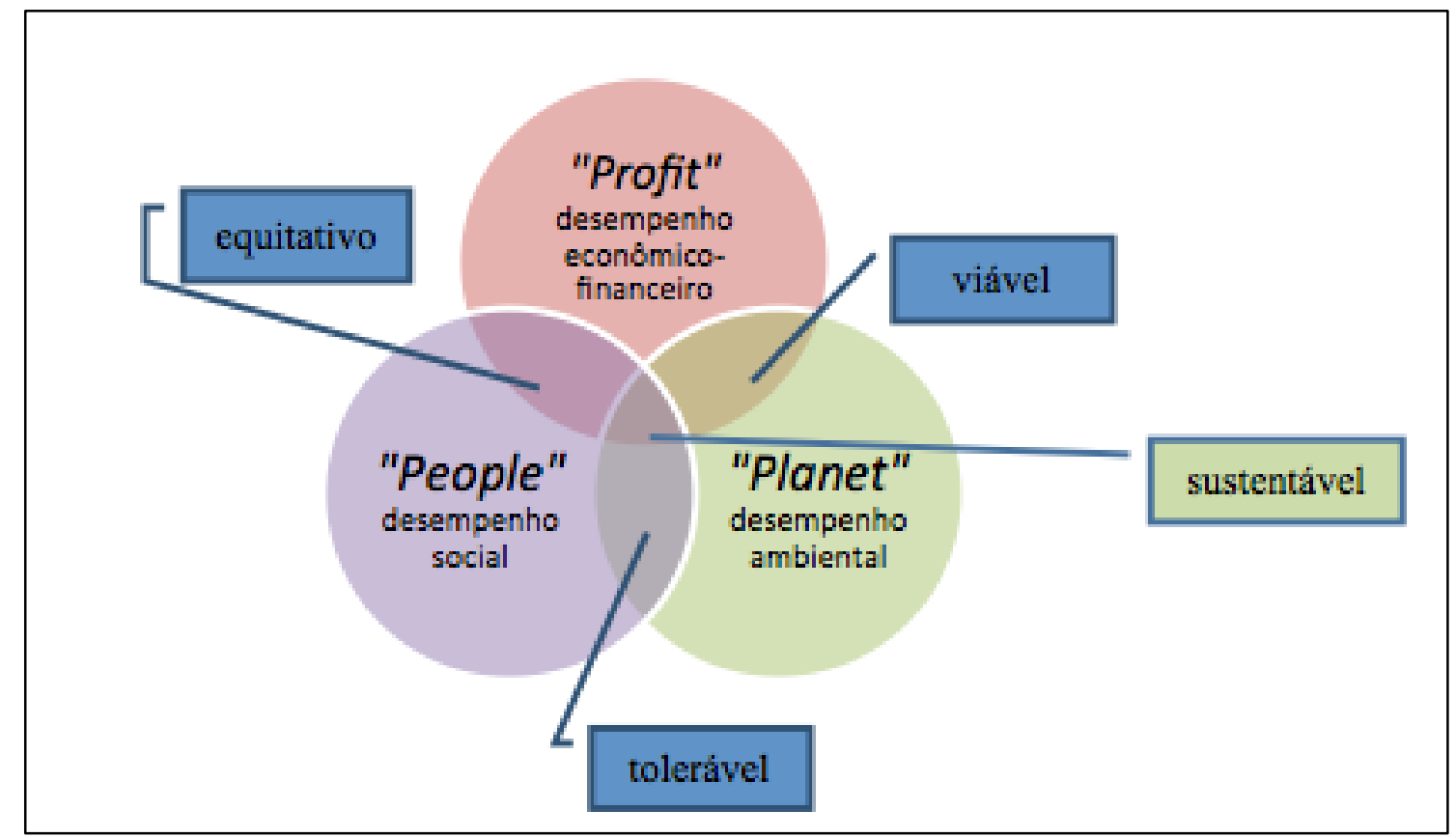

Figura 1 - A abordagem dos "3P's" de Elkington

Fonte: Elaboração própria, a partir de Elkington (2001).

Avaliar bons resultados de uma empresa no sentido do TBL - ou 3P's significa ponderar seus resultados para além dos acionistas, numa visão que poderíamos chamar de "capitalismo de stakeholders", que envolveria uma visão de negócios a partir de uma ética com relação às partes interessadas num sentido mais amplo, envolvendo funcionários ou colaboradores, os demais agentes ao longo da cadeia produtiva ou de agregação de valor - como fornecedores, clientes, e outros, grupos de interesse, sociedade civil organizada, vizinhos das plantas produtivas, e por aí adiante. É nesta senda que se colocam as iniciativas de responsabilidade social e ambiental ou socioambiental, de governança socioambiental, os relatórios que buscam dar publicidade aos resultados nas dimensões social e ambiental, dentre os quais os guias da Global Reporting Initiative - GRI - talvez seja a mais conhecida.

Desde o trabalho original de Porter e Van Der Linde (1995), no qual os autores propugnavam a oportunidade criada pela crescente onda da sustentabilidade para a implementação de estratégias win-win pelas empresas, existe toda uma literatura de gestão a respeito da sustentabilidade corporativa. $\mathrm{O}$ 
assunto tem sido abordado em suas variadas implicações, tais como no que tange à gestão de pessoas, inclusive suas demandas em termos de qualificação; no que diz respeito ao desenvolvimento de novos processos ou ao aperfeiçoamento daqueles pré-existentes com vista à melhoria de seus resultados para a dimensão ambiental ou à sua ecoeficiência; sua incorporação estratégica no sentido da concepção e desenvolvimento de novos produtos e serviços; no que tange a uma visão gerencial da inovação; e mesmo no que diz respeito à produção acadêmica nesse campo.

Recentemente, Porter \& Kramer (2011) reconhecem que

In recent years business increasingly has been viewed as a major cause of
social, environmental, and economic problems. Companies are widely
perceived to be prospering at the expense of the broader community. Even
worse, the more business has begun to embrace corporate responsibility,
the more it has been blamed for society's failures. The legitimacy of
business has fallen to levels not seen in recent history. (Porter \& Kramer,
2011, p. 1)

A razão central para esta perda de legitimidade é identificada pelos autores pela continuidade de práticas corporativas que se assentam numa compreensão equivocada do que vem a ser "valor": os resultados financeiros de curto prazo que deixam de lado as necessidades dos consumidores e ignoram as influências mais amplas que determinam o sucesso corporativo no longo prazo. Ao lado de um agudo, embora breve, diagnóstico da crise da legitimidade corporativa, os autores propõem uma visão menos restritiva e míope de valor ao mundo corporativo: o valor partilhado ou shared value. A observação que se pode fazer aqui é direta. O que os autores estão a propor diz respeito a uma gestão corporativa menos danosa ao meio ambiente e à sociedade e, por conseguinte, uma "gestão da legitimidade corporativa" - como já observamos em Godard (1993) e Corazza (2000, 2001 e 2003). Portanto, é panglossiana a ideia de que uma ampla incorporação da visão de shared value, assim como as políticas de responsabilidade socioambiental corporativas - como aquelas baseadas em alguma versão do TBL, possa ser o caminho para a sustentabilidade da existência humana da maneira que a temos conhecido.

É admirável que uma consideração crítica, e algo semelhante à nossa, da proposta de Porter \& Kramer (2011) venha justamente de Elkington (2012), que se mostra muito menos entusiasta a respeito do conceito shared value, identificando a capacidade do capitalismo em gerar valor justamente pela capacidade de "convert 
natural capital that has evolved over millions of years into things that financial markets value" (Elkington, 2012). O mesmo deveria ser dito com respeito ao trabalho humano, como se sabe.

Admirável, mas não surpreendente. A trajetória de Elkington é a de um sociólogo interessado na compreensão dos reveses sociais e ambientais do capitalismo. Seu livro traz uma visão que também pode ser tomada como panglossiana sobre as possibilidades de solução do problema da sustentabilidade social e ambiental pelas empresas ao identificar "sete revoluções" que estariam a desafiar e moldar novas práticas no mundo dos negócios (Elkington, 2001). Essas sete revoluções estariam relacionadas a transformações radicais nas formas de internalização da questão ambiental pelas empresas, na emergência do "capitalismo de stakeholders", numa maior transparência nos negócios, na responsabilidade ampliada do produtor, na mudança das relações entre estado-capital-sociedade, na importância da antecipação de mudanças e impactos, e na emergência de sistemas decisórios participativos.

Como não tomar como panglossiana a visão de Elkington (2001) sobre esse capitalismo de stakeholders? O autor realmente acredita que essas revoluções se processam na realidade? O que nos parece é que temos nesta obra um bom exercício de persuasão que, por vezes, nos parece também irônico. O autor emprega amplamente dados sobre acidentes catastróficos cuja origem está em práticas de grandes transnacionais. Nossa leitura das "revoluções" propostas por Elkington é a de "desafios". A estrutura do livro nos parece confirmar essa percepção. Por exemplo, na parte dois do livro, a que é voltada para a compreensão das sete revoluções, há uma estrutura interna que se reporta às mesmas questões: "por que se preocupar", "o que há de novo, gurus?", "visões dos comitês executivos", "o que está borbulhando", "vencedores, derrotados" e "chaves para o século XXI". O livro "clama" pela adesão das empresas a uma visão que busca a "civilização" e o "bom comportamento corporativo", baseada no valor fundamental da concorrência intercapitalistas. Por irônico que pareça, esse clamor está enunciado no próprio título do livro: "Cannibals with forks". Elkington, o sociólogo, vê as empresas crescendo, devorando-se mutuamente. Propondo a abordagem TBL, procura colocá-las civilizadamente ao redor da mesa, munidas de garfo e faca! 


\section{UMA CIÊNCIA, UM CAMPO INTERDISCIPLINAR OU UM PROGRAMA DE PESQUISA?}

Para muitos pesquisadores, que não se restringem a cientistas naturais como biólogos e ecólogos, incluindo também filósofos e historiadores do ambientalismo, a distinção entre sustentabilidade forte e fraca não faz sentido; também para eles, a falta de "hierarquia" nas três bottom lines da abordagem TBL é uma forte indicação de uma absoluta falta de percepção do caráter crítico dos serviços prestados pelos sistemas naturais. O economista diria que, para estes cientistas, a elasticidade de substituição entre capital natural e capital feito pelo homem é sempre extremamente baixa, pois, para eles, "in a world in which many species are obliterated will be one in which humans suffer too" (Neumeyer, 2010).

Esta é a perspectiva abraçada pela Ciência da Sustentabilidade. Alguns consideram este como um novo campo científico (como Schoolman et al, 2011 ou Nelson \& Vucetich, 2012). Para outros, trata-se de um programa de pesquisas emergente, também interdisciplinar (cf. Clark \& Dickson, 2003). A distinção entre campo científico e programa de pesquisa, em nossa perspectiva, não é o mais essencial. O reconhecimento da interdisciplinaridade, ao contrário, sim, pois deve reconhecer e enfrentar dificuldades epistemológicas e metodológicas profundas. Do ponto de vista epistemológico, seria possível questionar o objeto de estudo, o que traz de volta a problemática conceituação da sustentabilidade, que é o que nos interessa neste artigo. Do ponto de vista metodológico, uma questão absolutamente relevante - e, como é evidente, que depende da primeira - seria "como mensurar a sustentabilidade".

A interdisciplinaridade aparece como categoria unificadora das visões dentro da Ciência da Sustentabilidade. De acordo com Clark \& Dickson, "Sustainability Science focuses on the dynamic interactions between nature and society" e, a respeito do caráter controverso do termo, ponderam que:

"The term Sustainability Science has been controversial, connoting to some a mature discipline with shared conceptual and theoretical components that most certainly does not exist. One alternative descriptor is the science of sustainability', which conveys the notion of multiple sciences addressing a common theme. Our use of 'sustainability science', like that of the National Research Council, carries this last meaning". (Clark \& Dickson, 2003:8061). 
Nesses termos, a Ciência da Sustentabilidade conformaria uma arena interdisciplinar, onde contribuições das ciências naturais e sociais, da engenharia e da medicina, seriam amalgamadas no intuito de conformar um programa de pesquisa voltado ao avanço do conhecimento para o alcance da sustentabilidade ("a knowledge-driven agenda") e à solução de problemas ("a problem-driven agenda") (Clark \& Dickson, 2003).

Uma avaliação recente das publicações na temática da sustentabilidade feita por Bettencourt \& Kaurk (2011) parte justamente do reconhecimento da ambição da agenda da ciência da sustentabilidade em integrar a teoria, a "ciência aplicada" e as ações de policy para avaliar o esforço de síntese interdisciplinar dos estudos nesse domínio. Os autores empreenderam um estudo bibliométrico no qual analisaram a evolução temporal, a distribuição geográfica, a composição disciplinar e a estrutura de colaboração, a partir do qual evidenciam um crescimento explosivo de publicações, inclusive com novos periódicos voltados especificamente à temática da sustentabilidade e um crescimento do pool de novos autores, com interações progressivamente mais importantes.

Quanto à decomposição disciplinar, os autores evidenciam um padrão no qual um pequeno viés em favor das ciências sociais e policy em comparação às ciências biológicas. Há ainda contribuições em proporções equilibradas provenientes das engenharias (química, mecânica e civil), segundo puderam constatar os autores. No que diz respeito à "pegada geográfica", ou seja, a proveniência geográfica das autorias, os autores constatam um padrão que consideram incomum, com conexões entre cidades e nações de diferentes níveis de desenvolvimento. A decomposição das contribuições desses estudos entre as disciplinas tradicionais revela ênfase na gestão de sistemas humanos, sociais e ecológicos, vistos de uma perspectiva das engenharias e da policy.

Os autores ainda estudam a "coesão" desse campo interdisciplinar, aferida a partir das citações e das colaborações nos artigos publicados. De acordo com sua avaliação:

"[...] the field of sustainability science has become unified in terms of most authors belonging to the same large giant cluster of collaboration and citation. These networks span the world geographically and a wide range of disciplines in the social sciences, biology, and engineering, all primarily 
concerned with the integrated management of human, social, and biological systems" (Bettencourt \& Kaurk, 2011).

Os autores advertem que esse campo ainda é pouco investigado nessas dimensões. Aquilatar sua interdisciplinaridade ainda constitui um desafio. Os dados apresentados pelos autores, sobretudo com relação às redes de colaboração e de citações, instigam novas questões. É curioso verificar, por exemplo, o papel de Washington, DC, nessas redes. Pode também ser significativo, considerando a centralidade que Washington ainda desempenha nas estruturas decisórias da economia mundial. Mas este é apenas um estudo bibliométrico. Outras análises seguramente são necessárias.

Ainda assim, é interessante que este levantamento amplo e a análise dos estudos publicados desde a década de 1980 na temática da sustentabilidade, com a constatação da emergência de um componente gigante de colaboração científica, tenha permitido aos autores concluir que existe uma progressiva integração das abordagens disciplinares tradicionais. Na sua interpretação, existe um "campo da sustentabilidade", uma área científica jovem e crescente, que se se caracterizaria por uma prática científica incomum, inclusiva e ubíqua, o que, na avaliação desses autores, pode ser um bom augúrio para seu impacto e para sua longevidade.

\section{CONSIDERAÇÕES FINAIS}

Vivemos um momento de forte integração do conceito de sustentabilidade pelas agendas pública e privada. A guiar essa integração, estão as questões que se tornam emergenciais em nossos dias no que tange às possibilidades futuras da civilização. As mudanças climáticas globais são as que se colocam de maneira mais dramática. Ao lado dela, a taxa de perda da biodiversidade, os ciclos do nitrogênio e do fósforo, a depleção do ozônio estratosférico, a acidificação dos oceanos, a pressão sobre o uso de água doce, as mudanças no uso da terra, a carga de aerossol na atmosfera e a contaminação química são apontadas por Rockstrom et al (2009) como as "nove fronteiras planetárias", que identificam o desafio de manter a estabilidade das condições de vida que tem até aqui caracterizado nosso período geológico. 
"Sustentabilidade" tem a ver com o bem-estar da humanidade no futuro; portanto, tem a ver com a preservação da estabilidade dessas "fronteiras planetárias". Apresentamos, neste artigo, três visões distintas do conceito de sustentabilidade. Em primeiro lugar, aquela oferecida pelas Ciências Econômicas, em suas acepções de sustentabilidade forte e fraca. Em segundo lugar, a desenvolvida no âmbito da gestão e que representa a visão provavelmente mais difundida pela literatura em sustentabilidade: a visão da Triple Bottom Line, também conhecida como "3Ps" ou "três pilares da sustentabilidade". Finalmente, apresentamos a concepção que identifica a emergência de uma Ciência da Sustentabilidade, como um programa de pesquisa ou como um campo interdisciplinar.

Cabe, em nossa perspectiva, tentar compreender de que maneira elas podem nos esclarecer sobre quais seriam os caminhos a seguir. Há um novo conceito de desenvolvimento por se construir. Essas visões podem nos auxiliar a avaliar os caminhos do desenvolvimento? Podem nos ajudar a examinar as iniciativas em Ciência, Tecnologia e Inovação?

Temos visto que, a fim de aquilatar a medida em que as práticas empresariais contribuem para a melhoria da "triple bottom line", foram desenvolvidas metodologias de avaliação, como aquelas das guidelines da Global Responsibility Initiative e do Dow Jones Sustainability Index, dentre outros. Nesses casos, como em outras metodologias análogas, o sentido da avaliação é bottom-up, ou seja, parte-se das iniciativas analisadas (negócios, projetos ou tecnologias por exemplo) tendo como base da avaliação (baseline) os estados anteriores dessas iniciativas (ou da situação afetada pela iniciativa em um momento anterior a sua realização, em suas dimensões ambiental, social e econômica.

Algumas críticas que se interpõem a essa abordagem de avaliação da sustentabilidade guardam correspondência com o que vínhamos a expor com respeito à perspectiva da triple bottom line da sustentabilidade. É possível compreender essas críticas ao modelo conceitual de sustentabilidade proveniente da abordagem da TBL (e às metodologias de avaliação da sustentabilidade apoiadas nesse "modelo") a partir de dois pontos sistematizados aqui a partir de Gibson (2001) e Pope et al (2004).

O primeiro ponto se refere ao viés de status quo, que se relaciona ao fato de que a visão TBL não parte de uma situação geral desejável. Partir de um estado ideal 
para as três dimensões implicaria uma visão top-down que teria como "ponto de partida" estados desejáveis dos indicadores de sustentabilidade para aquelas três dimensões, coisa que não poderia ser substituída pela simples consideração de benchmarks. O segundo ponto de crítica é de natureza epistemológica e aponta para o fato de que a abordagem TBL reflete a clivagem disciplinar moderna enquanto que uma perspectiva sistêmica da sustentabilidade ensejaria uma perspectiva holística.

Ao ponderar sobre esses dois pontos críticos, os autores sugerem, como alternativa trabalhar com "estados desejáveis" por meio da explicitação de um conjunto de "critérios de sustentabilidade" (que serão específicos para cada caso" para cada "princípio de sustentabilidade", conforme enunciados pela Declaração do Rio. Essa direção proposta por Gibson (2001) e Pope et al (2004) implica que se estabeleçam "rumos" para as mudanças sociais, institucionais e tecnológicas. Portanto, o que propõem é uma abordagem normativa que, segundo nossa percepção, se coloca para além das clivagens disciplinares que estão na base da TBL. Tendo em vista o estado incipiente dos estudos da Ciência da Sustentabilidade, os esforços para a compreensão do que temos chamado, em geral tão frouxamente, de sustentabilidade e das possibilidades futuras para a humanidade não poderiam ser mais urgentes.

\section{AGRADECIMENTOS}

Rosana I. Corazza e Maria Beatriz M. Bonacelli agradecem aos colegas que, entre 2010 e 2012, participaram intensamente dos estudos e debates sobre inovação e sustentabilidade no âmbito das atividades do GEOPI - Grupo de Estudos sobre Organização da Pesquisa e da Inovação, do DPCT-IG/Unicamp e do Fórum Permanente sobre os Desafios da Avaliação da Sustentabilidade na FCA/UNICAMP: Flávio Arantes, Adriana Bin, Luíza Capanema, Ana Maria Carneiro, Fernando Colugnati, Paula Drummond de Castro, Ana Flávia Ferro, André T. Furtado, Carolina Mattos e Sergio Salles-Filho. Eventuais erros e omissões são da exclusiva responsabilidade dos autores. Rosana Corazza agradece à Fundação de Amparo à Pesquisa do Estado de São Paulo - FAPESP, pelo financiamento (Processo 09/16647-8). 


\section{REFERÊNCIAS}

AYTES, R. U.; VAN DEN BERGH, J. C. J. M.; GOWDY, J. M. Viewpoint: weak versus strong sustainability, 1998. Disponível em

<http://kisi.deu.edu.tr/sedef.akgungor/ayres.pdf>. Consultado em: setembro de 2012.

BETTENCOURT, L. M. A.; KAURC, J. Sustainability Science, Sustainability Science: evolution and structure of sustainability science. Proc Natl Acad Sci U S A. 108(49): 19540-19545, 2011. Disponível em:

<http://www.ncbi.nlm.nih.gov/pmc/articles/PMC3241817/>. Acesso em junho de 2013.

CLARK, W. C.; DICKSON, M. N. Sustainability science: the emerging research program. Proc Natl Acad Sci U S A. 100(14):8059-8061, 2003. Disponível em: <http://www.ncbi.nlm.nih.gov/pmc/articles/PMC166181/>. Consultado em junho de 2012.

CMMAD/ONU. Nosso Futuro Comum. Rio de Janeiro: Ed. Fundação Getúlio Vargas, 2001 [1987].

ELKINGTON, J. Canibais com garfo e faca. São Paulo: Makron Books, 2001 [1997].

ELKINGTON, J. Sustainability should not be consigned to history by Shared Value. Sustainable Business Guardian Blog, 2012. Disponível em:

$<$ http://www.guardian.co.uk/sustainable-business/sustainability-with-johnelkington/shared-value-john-elkington-sustainability>. Acesso em maio de 2013

HOTELLING, H. The economics of exhaustible resources. Journal of Political Economy, 39:137-175, 1931.

JABBOUR, C. J. C.; JABBOUR, A. B. S. L.; OLIVEIRA, J. H. C. The Perception of Brazilian Researchers concerning the Factors that Influence the Citation of their Articles: A Study in the Field of Sustainability. Serials Review, 39(2):93-96, 2013.

KEMP, R. Las tecnologías sostenibles no existem! Ekomiaz. Revista Vasca de Economia: 22-39, 2010. Disponível em: <http://www1.euskadi.net/ekonomiaz/taula4_c.apl?REG=1081>. Consultado em agosto de 2011.

MORRISON-SAUNDERS, A. \& THÉRIVEL, R. Sustainability Integration and Assessment. Journal of Environmental Assessment, Policy \& Management, 8(3): 281-298, 2006.

PIGOU, A. C. Economics of Welfare. London: Macmillan, 1920.

POPE, J., ANNANDALE, D.; MORRISON-SAUNDERS, A. Conceptualising Sustainability Assessment. Environmental Impact Assessment Review, 24: 595-616, 2004.

PORTER, M. E.; KRAMER, M. R. The Big Idea: Creating Shared Value, Harvard Business Review, 2009. Disponível em: <http://www.professoralanross.com/wpcontent/uploads/2011/03/The-Big-Idea_-Creating-Shared-Value-Harvard-BusinessReview.pdf>. Acesso em abril de 2013. 